\title{
Morfologia urbana e a revolução dos dados
}

\author{
Romulo Krafta $^{\mathrm{a}}{ }^{(}$, Alice Rauber $^{\mathrm{b}}$ \\ ${ }^{a}$ Universidade Federal do Rio Grande do Sul, Departamento de Urbanismo, Programa de Pós-Graduação \\ em Planejamento Urbano e Regional, Porto Alegre, RS, Brasil. E-mail:krafta@ufrgs.br \\ ${ }^{\text {b } U n i v e r s i d a d e ~ F e d e r a l ~ d o ~ R i o ~ G r a n d e ~ d o ~ S u l, ~ D e p a r t a m e n t o ~ d e ~ U r b a n i s m o, ~ P r o g r a m a ~ d e ~ P o ́ s-G r a d u a c ̧ a ̃ o ~}$ \\ em Planejamento Urbano e Regional, Porto Alegre, RS, Brasil. E-mail: alicerauber@hotmail.com
}

https://doi.org/10.47235/rmu.v8i1.151

Submetido em 5 de junho de 20201. Aceito em 29 de junho de 2020.

\begin{abstract}
Resumo. Morfologia Urbana é um campo altamente dependente de dados espaciais urbanos. Sua dificuldade de obtenção constituiu durante muito tempo obstáculo ao desenvolvimento de pesquisas na área, porém, mais recentemente, o aumento na disponibilidade de dados, a emergência do big data e o crescente desenvolvimento de ferramentas e tecnologias de informação, vem abrindo novas possibilidades para explorar digitalmente $o$ território. Não se trata apenas de disponibilidade abundante de dados e softwares para manipulá-los, mas novas capacidades de integrá-los, o que encoraja a cruzar fronteiras disciplinares. $O$ presente artigo tem como foco a relação entre o campo da morfologia urbana, caracterizado por contornos bem definidos em termos de quais dados são requeridos, e o que tem sido feito na dita fronteira do conhecimento, onde dados de diferentes tipos são combinados. Como contribuição este trabalho identifica perspectivas para futuras pesquisas, mostrando como a morfologia urbana pode se beneficiar dessa revolução dos dados, em especial utilizando-se de novos mecanismos descritivos e analíticos. Isso é evidenciado através da exploração de grafos temporais, hipergrafos e grafos multicamadas, ainda pouco empregada nos estudos urbanos, mas que demonstra potencial para: a) descrições multidimensionais da forma urbana; b) explorações de análises do espaçotempo.
\end{abstract}

Palavras-chave. morfologia urbana, big data, redes multicamadas, redes temporais

\section{Introdução}

Por muito tempo, na verdade desde os momentos iniciais da ciência urbana, a indisponibilidade de dados sobre as diferentes realidades urbanas constituiu um dos principais obstáculos ao seu desenvolvimento. Seja para alimentar longos e minuciosos procedimentos de classificação de componentes e atividades, seja para testar hipóteses deduzidas mediante elaboração intelectual abstrata, a tarefa de coletar, registrar, estruturar e disponibilizar dados suficientes para fundar análises quantitativas sempre pareceu maior, mais difícil, cara e demorada dentre todas as possíveis atividades de pesquisa urbana. Problemas relativos a tipo, formato, agregação, durabilidade, delimitação geográfica, compatibilidade, atualidade, combinados com técnicas particulares de coleta e registro, escala espacial e temporal, tudo tratado de forma setorial e localmente diferenciadas terminaram por constituir uma babel de dados desencontrados e descontinuados em cada cidade. O esforço para realizar essas tarefas geralmente consumiu a quase totalidade do tempo e recursos das eventuais equipes técnicas dos órgãos técnicos municipais, relegando para um plano secundário e subdesenvolvido as tarefas analíticas propriamente ditas e suas subsequentes projeções, explorações e formulações teóricas e metodológicas. Em resumo, o planejamento urbano tem se resumido a coletar dados e, com algum esforço, reconhecer algum tipo de padrão 
socioespacial mais visível. Eventuais planos, programas e projetos teriam fatalmente uma relação débil e meramente conjectural àqueles dados e padrões.

A evolução dos chamados modelos urbanos quantitativos integrados elevou esse problema a um novo patamar ao buscar articular diferentes variáveis do sistema urbano - distribuição espacial de população, emprego, consumo e equipamentos, padrões de fluxo, infraestrutura, insumos externos, trocas, evolução demográfica, econômica e ambiental, e o que seja. Embora desde o ponto de vista teórico e metodológico isso tudo pudesse ser compatibilizado num sistema de equações, na prática determinou a virtual impossibilidade de haver continuidade entre pesquisa científica e atividade técnica aplicada. Tudo isso por causa dos dados, ou de sua ausência. Obviamente esse quadro nunca foi homogeneamente caótico: diferentes países, diferentes cidades desenvolveram, com maior ou menos rigor e sucesso, procedimentos de homogeneização de bases de dados, tanto a nível local (prefeituras, órgãos metropolitanos e regionais) quanto nacional (no Brasil, reconhecimento ao trabalho do IBGE, sem dúvida).

Entre os campos com alta dependência a dados, encontra-se a Morfologia Urbana, que surgiu como um nicho dos estudos urbanos baseado fundamentalmente em classificação e análise empírica. Nossas indagações fundamentais eram quanto à natureza, emergência e evolução de padrões de forma, num plano mais operativo, e quanto à abrangência, isto é, à possibilidade de se construir uma explanação autônoma do fenômeno urbano a partir de sua morfologia, no plano teórico. Tendo base estritamente empírica, não seria alheia às vicissitudes da busca de dados, mas, por outro lado, sendo, digamos, meticulosa quanto a natureza e tipo de dados, teria uma chance. Aparentemente, foi o que aconteceu: uma redução do número de variáveis que permite manter uma escala compatível com o fenômeno (exemplo: o mapa axial) e/ou uma redução da escala que permita considerar a suposta riqueza da forma construída (exemplo: os estudos tipológicos). O mapa axial promoveu uma devastação na população de possíveis variáveis explanatórias da forma urbana ao reduzir o "problema" a um conjunto de linhas retas que descrevem o sistema viário urbano segundo uma configuração, ou seja, um sistema de linhas posicionadas umas em relação às outras, livre de escala. Essa configuração supostamente deteria algumas propriedades essenciais da forma urbana, reveladas, então, através de medidas de hierarquia espacial, como acessibilidade (ou integração), centralidade (betweenness) ou controle.

Se mapa axial é provavelmente a maneira mais sumária e econômica de descrever o sistema urbano, na outra extremidade da escala de observação os estudos tipológicos mergulham na busca da diferenciação fina da forma construída, tanto no plano espacial (diversidade) quanto temporal (evolução). Essas duas dimensões, imitando a Física, jamais se encontraram, com o quê o campo da Morfologia Urbana permanece oscilando entre duas correntes teóricas, uma de base relativista - fundada na configuração espacial, e outra de base atômica - fundada na classificação tipológica.

O presente artigo discute como que a revolução digital e o advento do fenômeno big data vêm impactando o campo da Morfologia Urbana. Na primeira parte apresentamos como essa enorme disponibilidade de dados beneficia diretamente todas as vertentes da Morfologia Urbana, retomando seus usuais mecanismos descritivos e analíticos. Em seguida mostramos possíveis expansões para as bases descritivas usualmente utilizadas na chamada abordagem configuracional, que mediante a combinação de dados de diferentes tipos, abrem possibilidadesde de integração com outras abordagens. Por fim, indicamos como que a Morfologia Urbana, com base em uma abordagem de grafos multicamadas, pode evoluir das análises do espaço para análises do espaço-tempo, passando de leituras de estado para leituras de processo.

\section{O tsunami de dados}

O incrível desenvolvimento da tecnologia da informação mudou radicalmente o quadro de referência dos estudos urbanos em geral e da morfologia urbana em particular. Quatro parecem ter sido os vetores principais de mudança, que vêm provocando uma verdadeira revolução dos dados. O primeiro se refere à passagem de bases analógicas para digitais dos dados usualmente coletados e mantidos por organismos de planejamento e gestão de cidades. A progressiva 
digitalização dos dados urbanos propiciou, por sua vez, dois novos processos, a saber, a disponibilização via internet e a normalização de escalas, tipos, níveis de agregação, classes, etc., via geoprocessamento e sensoriamento remoto.

Bases de dados de muitas cidades passaram a ser normalizadas e compatíveis entre si e acessíveis publicamente. Pode-se referir a esse tipo de dado como dedicado, quer dizer, conjuntos de dados coletados e mantidos com vistas a alimentar processos de análise, exploração e tomada de decisão particulares. Como tal, teriam sido construídos com métodos e procedimentos reconhecidos e minimamente acreditados.

O segundo vetor é constituído pelo incrível acervo Google - Earth e Maps, que cobre o globo e disponibiliza imagens de alta resolução de virtualmente toda aglomeração urbana existente, em três dimensões e ainda passíveis de serem 'percorridas' e verificadas prédio a prédio. As pessoas dedicadas ao estudo da forma urbana jamais poderão ser gratas o suficiente por isso. A despeito de suas qualidades, a plataforma Google é de formato proprietário, impondo restrições de uso e acesso aos dados que podem limitar o uso em pesquisas (Boeing, 2018). Assim, a iniciativa Open Street Map, semelhante ao Google quanto ao caráter global, mas que se diferencia por ser um projeto de mapeamento colaborativo que disponibiliza dados abertos, tem sido uma das principais fontes para obter bases geográficas, especialmente do sistema viário e seus atributos e seus atributos - tipo de via, nome, velocidade, número de pistas.

$\mathrm{O}$ terceiro vetor somente existe em decorrência do anterior, se valendo deste para acumular informação adicionada pelos usuários, seja diretamente (fotos de lugares, preferências, localizações, etc.) registradas diretamente nos mapas e fotos orbitais, seja indiretamente (localização de negócios, serviços e outros elementos de interesse) mediante acesso pago, no caso do Google, ou gratuito, no caso do OSM. Este seria um tipo de dado diferente do primeiro, no sentido de não obedecerem a métodos e procedimentos uniformes.

Por fim, o quarto vetor se refere a dados gerados de forma mais passiva, através de celulares, redes sociais, aplicativos, cartões e serviços. Através desses, é possível acessar informações - em tempo real, às vezes sobre localização, movimento e comportamento dos indivíduos. Tomados os devidos cuidados com questões de privacidade, esse tipo de dado tem potencial enorme para estudar padrões de comportamento de massa.

$\mathrm{O}$ termo big data se refere à disponibilidade de enorme quantidade de dados que podem facilmente ser processados por computador. O potencial de uso em pesquisas é enorme, porém traz a necessidade de desenvolvimento de novos métodos para manipular e analisar esses dados, que, muitas vezes, não são gerados para essa finalidade (Offenhuber e Ratti, 2014). Dados de telefonia móvel (Greco, 2014) ou de redes sociais (Netto et al., 2017), por exemplo, desde que manipulados e filtrados, podem fornecer uma visão sobre os deslocamentos das pessoas na cidade, algo que anteriormente só era possível através de caras pesquisas origemdestino ou trabalhosas verificações in loco.

Outro exemplo do potencial que big data representa diz respeito à obtenção de bases vetoriais de sistema viário, nesse caso de grande interesse dos cientstas de redes. Através de procedimentos simples, como o pacote OSMnx, desenvolvido por Boeing (2017), é possível obter mapas de segmentos de rua ou de intersecções viárias para qualquer recorte espacial desejado, praticamente de qualquer parte do mundo. Comparar uma grande quantidade de cidades, alguns anos atrás, envolveria um demorado e trabalhoso processo de desenho e coleta de dados, mas atual facilidade de obtenção de bases do OSM permite, hoje, estudos cada vez mais robustos sobre verificação de padrões universais, observáveis a partir de grandes amostras de cidades (Boeing, 2018; Kirkley et al., 2018).

\section{Morfologia urbana e sua relação com big data}

Seria de se esperar que, dentre as diversas vertentes de estudos sobre a cidade, a de morfologia urbana tenha sido a maior beneficiária da avalanche de novos dados, particularmente fotos orbitais de alta resolução, mapas, street-view, em primeiro lugar, e mesmo dados complementares também ali contidos, como usos pontuais do solo, equipamentos, descrição de intensidade de fluxos, quantidade comparativa de fotos de lugares, postadas por usuários, preferências por lugares e percursos capturadas por GPS ou por 'likes', 
distribuição de usuários capturada por sinal de celular, mapas colaborativos, etc.

Para entender as possibilidades de apropriação desse novo manancial de dados sobre cidades e vida urbana pela área de morfologia urbana é necessário explicitar seus usuais mecanismos descritivos e analíticos, buscando identificar quê dados são demandados, como se processam as descrições e análises e, como consequência, quais dados agora disponíveis as alimentariam, sob que condições. Num universo de grande diversidade, tanto de componentes (formas construídas, partições fundiárias, espaços públicos) quanto de composições (tecidos urbanos) a investigação da forma urbana busca identificar padrões, quer dizer, regularidades na distribuição, ordenamento ou estruturação desses componentes, seja na dimensão espacial ou temporal.

Distribuição espacial implica descrever (identificar e diferenciar componentes e localizações) e analisá-los por proximidade, densidade, combinação, fazendo emergir eventuais padrões de regularidade, aglomeração, granulosidade, justaposição, complexidade, etc. Fotos orbitais de alta definição, combinadas com recursos de navegação (streetview), certamente constituem um repositório virtualmente inesgotável de dados para este tipo de descrição e análise, pelo qual devemos ser gratos (thanks, Google). Distribuição evolutiva, ou seja, distribuição espacial ao longo do tempo, desta vez focada na permanência e mudança de padrões distributivos dos componentes da forma urbana, não é ainda inteiramente satisfatória, visto que as séries temporais de imagens ainda são pequenas; não obstante o futuro disso parece muitíssimo promissor.

Investigação de padrões de ordenamento morfológico envolve procedimentos bem mais elaborados. Em primeiro lugar, identificação e diferenciação de componentes da forma urbana vai mais longe que a simples classificação segundo propriedades geométricas dos componentes, sejam eles bi ou tridimensionais normalmente aplicada na análise da distribuição. Aqui a noção de tipo e a consequente classificação tipológica dos componentes tende a mergulhar na história, na organização interna e no projeto de prédios e espaços públicos, envolvendo vários outros atributos tanto de cada componente, como também de suas articulações com outros na formação do tecido urbano propriamente dito. Nessa perspectiva, descrição de edificações e espaços abertos articulados, quer dizer, compostos de alguma maneira a gerar ordem, torna-se matéria central. Percebe-se que aqui as fotos orbitais navegáveis cumprem apenas uma parte da demanda por informação relevante. Na verdade podem constituir uma boa base inicial, a partir da qual a agregação de dados históricos virá fazer o real trabalho de reconstrução da gênese da forma urbana.

Estudos configuracionais destinam-se a revelar a estrutura da forma urbana; estrutura, no caso, pode ser entendida como o conjunto de relações vinculantes entre cada componente com todos os demais que formam a interdependência, o sistema espacial. Configuração espacial urbana envolve descrição de componentes - espaços abertos e formas construídas, e suas relações de adjacência, resultando num grafo de grandes proporções que pode, não obstante, ser medido segundo atributos hierárquicos. Diferentes medidas de centralidade irão revelar a estrutura espacial segundo diferentes critérios - grau de dependência do sistema em relação a cada um dos componentes, grau de intensidade urbana resultante da interação entre todos os componentes, posição relativa de cada componente, distância relativa, etc. Uma vez mais as bases de dados providos por repositórios de dados vetoriais e por fotos orbitais zoomíveis e navegáveis permitem agora que descrições e análises deste tipo sejam levadas a cabo mais facilmente, mesmo que para isso haja eventual necessidade de transformar dados 'brutos' em informação mais refinada, mediante procedimentos digitais disponíveis.

Como se vê, apenas parte relativamente pequena, apesar de altamente relevante, da chamada big data tem na verdade beneficiado e impulsionado a investigação da morfologia urbana, pela simples razão que essa maior parte não utilizada se refere a dados sobre o digamos software urbano: agentes, ações, movimentos, interações de ' $n$ ' naturezas e escalas, muitos deles escravos da forma urbana, quer dizer, realizadas no interior de um casco urbano dado e inelástico no curto prazo, mas outras tantas voltadas a 
adaptar esse casco às continuamente novas demandas de uso.

\section{Morfologia e análise do espaço}

Morfologia Urbana firmou-se no campo dos estudos urbanos praticando um controle rigoroso dos limites disciplinares e escolha de variáveis e métodos de investigação. Pioneiros desafiaram-se a produzir enunciados explanatórios do fenômeno urbano independentes das usuais variáveis socioeconômicas que, não obstante, fossem congruentes com enunciados oferecidos por outras áreas da pesquisa sobre cidades. Enunciados teóricos em morfologia urbana tenderam, nos seus primórdios, a se ater a descrições histórico-geográficas da evolução das cidades (morfogênese), como em Conzen (2004), descrições histórico-tipológicas da evolução de edificações e espaços públicos, como em Caniggia (1984) ou Panerai et al. (1997); estabelecendo paralelos morfológicos à história social, econômica e política das sociedades respectivas. Enunciados teóricos alternativos foram derivados de análise configuracional (denominados modelos de centralidade), cujas descrições se mostraram congruentes com aspectos do funcionamento (padrão genérico de fluxos, por exemplo) e de organização interna (padrão de uso do solo, valor da terra, intensidade de atividade) de cidades respectivas (Batty e Longley,1988; Krafta, 1994; Spinelli e Krafta, 1998).

Análise quantitativa do espaço urbano, da qual a chamada abordagem configuracional é parte, desenvolveu-se enormemente nos últimos anos, sugerindo que descrições, análises e eventualmente simulações da dinâmica urbana podem ser levadas a efeito a partir de uma base espacial, quer dizer, construídas a partir de uma base descritiva morfológica, adicionando variáveis denotativas de outros campos, como economia, infraestrutura, interação espacial e transportes, uso do solo, interação social, etc. Qualquer um desses setores, uma vez associado a uma base descritiva espacial adequada pode potencialmente produzir conhecimento mais abrangente do que isoladamente. Caberia aos interessados em morfologia urbana promover, mediante admissão de variáveis de natureza socioeconômica, aproximação às demais áreas da ciência urbana, tal como tem sido levado a cabo em diferentes centros de pesquisa urbana ${ }^{2}$.

\section{Abordagem configuracional e possíveis expansões}

A representação fundamental de um sistema espacial, ou morfológico, nesta denominada abordagem configuracional é um grafo, no qual vértices correspondem a unidades de espaço público e arestas a adjacências físicas. $\mathrm{Na}$ Figura 1 pode-se ver como um grafo desse tipo é produzido, usando as partições do espaço público em unidades mais utilizadas: linhas axiais, segmentos, e nós.

Representar os elementos que compõem a cidade e suas relações através da linguagem de grafos, trouxe ao campo da morfologia urbana a possibilidade de adaptar métodos clássicos da ciência das redes, como as medidas de centralidade citadas anteriormente. No entanto, é preciso reconhecer que a representação sob a forma de grafos simples, como vem sendo feita até agora, possui limitações. Alguns autores (Aleta; Moreno, 2018; Kivelä et al., 2014) sugerem que os grafos simples podem ser um obstáculo à representação de alguns fenômenos, ao olharem apenas para um tipo de relação de cada vez. Nicosia et al. (2013) argumentam que uma rede complexa raramente é isolada, sendo que muitas vezes alguns de seus nós poderiam ser parte de vários grafos ao mesmo tempo. Assim, observa-se, entre os cientistas de redes, crescente interesse por uma perspectiva de grafos multicamadas, que tende a ser uma representação mais realista de sistemas complexos, ao levar em conta múltiplos tipos de elementos e de relações (Kivelä et al., 2014; Nicosia et al., 2013). 

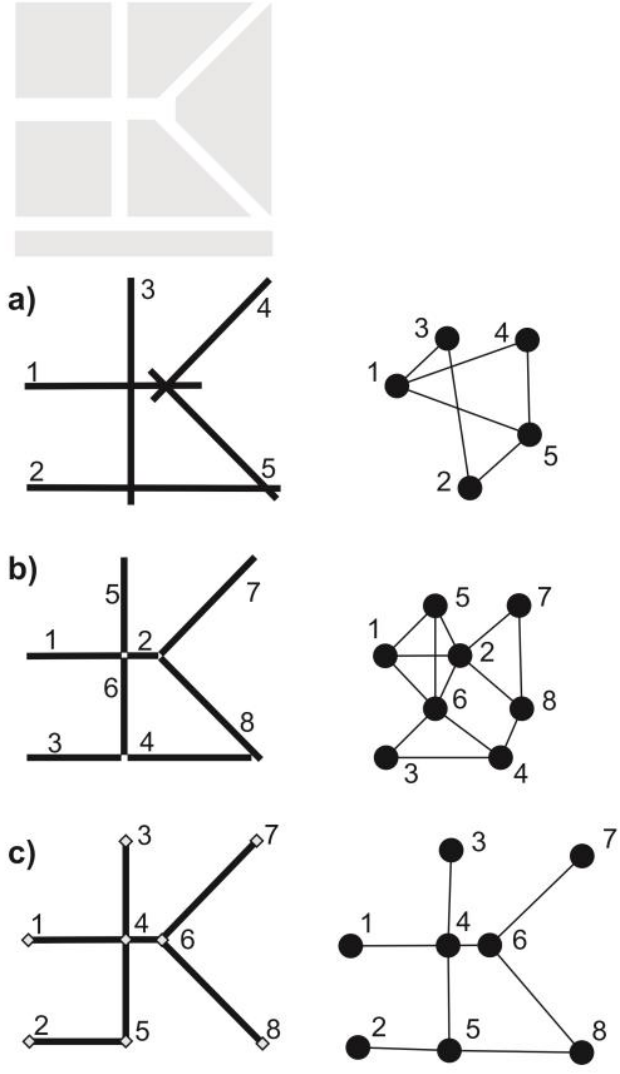

Figura 1. Sistemas descritivos dos espaços públicos por: (a) linhas axiais, (b) segmentos e (c) nós; e seus respectivos grafos (fonte: elaborada pelos autores).

As propriedades estruturais de certas redes e sua evolução, podem depender, de modo nãotrivial, de outra rede à qual esteja conectada ou de múltiplos tipos de relações entre os elementos. Esse é o caso das redes de transportes, redes sociais, redes de relações econômicas entre países e o próprio cérebro humano, onde cada rede é parte de um sistema maior, no qual um conjunto de redes interdependentes, com diferentes estruturas e funções, coexistem, interagem e evoluem. Consequentemente, tais sistemas poderiam ser melhor representados por grafos compostos por múltiplas camadas. Esse parece ser o caso também das cidades, sob uma perspectiva de sistemas complexos.

Uma cidade pode ser pensada como um grande sistema composto por subsistemas, que, por sua vez, também são compostos por subsistemas (Johnson, 2012). Isso remete à visão apresentada, décadas atrás, por Alexander (2015) em A city is not a tree, que enfatiza $o$ aspecto de rede presente nas cidades e os subsistemas que se sobrepõem. Conforme o autor, a cidade não pode ser considerada uma estrutura totalmente hierárquica - uma "árvore" - mas sim uma semitrama (semilattice), que é uma estrutura complexa, com sobreposições e ambiguidades. A cidade é cheia dessas estruturas sobrepostas e ambíguas, que são responsáveis por sua riqueza e complexidade. As tais estruturas em semitrama às quais Alexander se refere são, na verdade, princípios de organização, ou seja, são estruturas abstratas, e não formas físicas, conforme bem colocado por Porta $e t$ al.(2015). Uma abordagem baseada em grafos multicamadas, em que vértices e arestas representam elementos e relações de variados tipos, abre a possibilidade de operacionalizar a representação dessas distintas redes e estruturas que se sobrepõem na cidade. Tal abordagem, no entanto, ainda é pouco desenvolvida nos estudos de morfologia urbana, que até o momento têm se concentrado mais na representação da rede de espaços públicos, ao passo que outras variáveis costumam ficar de fora.

A seguir apontamos algumas alternativas para se pensar em aplicações de grafos multicamadas para o campo da morfologia urbana. Isso passa pela expansão da capacidade de representar o sistema espacial urbano, isto é, pela inclusão de novas variáveis à rede de espaços públicos interconectados, tradicionalmente representada por grafos simples na abordagem configuracional. Outras camadas, agora contendo dados externos, porém acopláveis à base espacial, podem ser adicionadas, conforme sugere a Figura 2. Para cada camada é preciso pensar em estratégias de representação, definindo critérios e explicitando o que são os vértices e as arestas. A chamada fase de modelagem de rede, destacada por Marshall et al. (2018), em que se toma esse tipo de decisão, é fundamental nesse caso. 


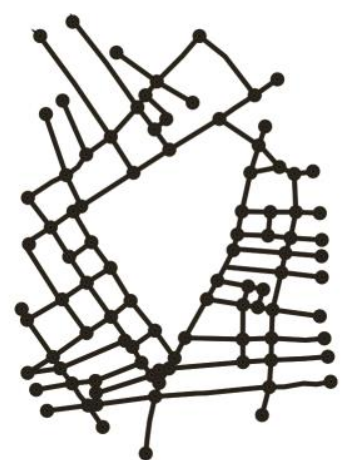

ESPAÇO PÚBLICO

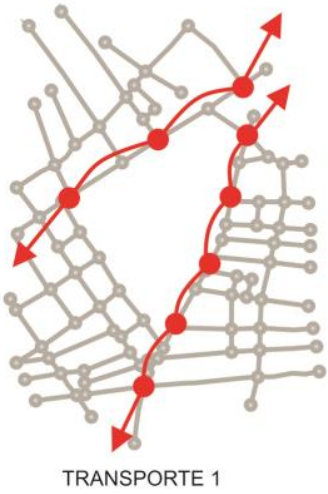

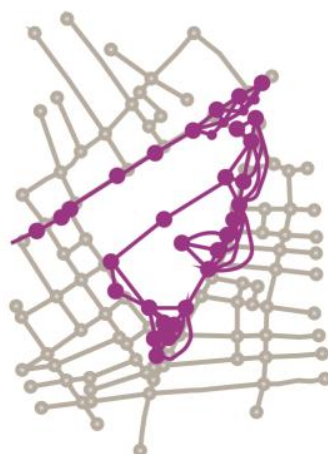

ORDEM SIMBÓLICA

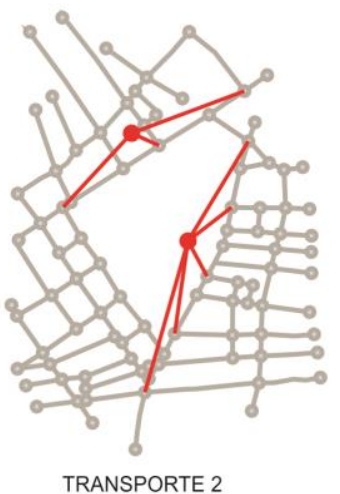

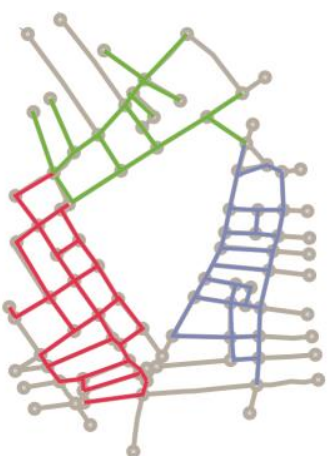

AFINIDADE TIPOLÓGICA

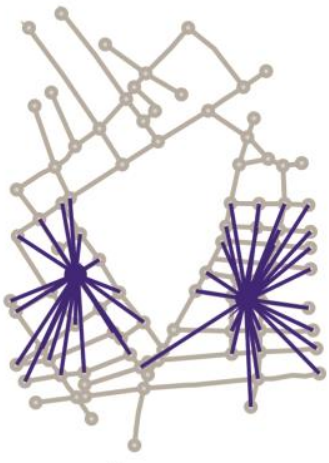

DEPENDÊNCIA FUNCIONAL

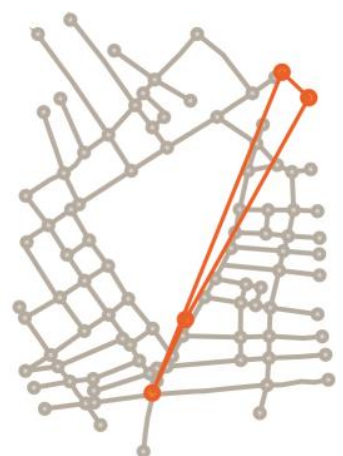

AFINIDADE COGNITIVA

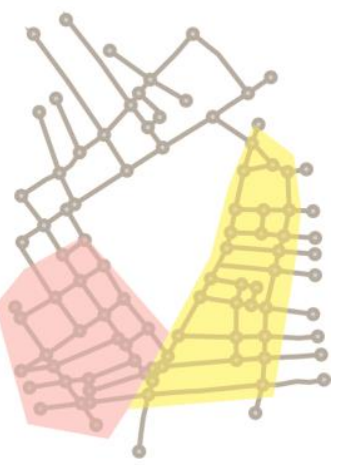

RENDA/ DENSIDADE

Figura 2. Possíveis camadas do grafo (fonte: elaborada pelos autores).

Quadro 1. Quadro multidimensional para representação em múltiplas camadas (fonte: elaborado pelos autores).

\section{Elementos}

DIMENSÃO ESPACIAL

Espaços públicos

\section{Estratégias de representação}

\section{a) Grafos simples;}

b) Definição de raios para processamento das medidas; c) Definição de valores de impedância nas arestas

\begin{tabular}{lc}
\hline DIMENSÃO & $\begin{array}{c}\text { Formas construídas } \\
\text { OBJETUAL }\end{array}$ \\
& Lipos edilícios \\
& Parcelas \\
\hline & Uso do solo \\
DIMENSÃO & Atividades \\
FUNCIONAL & Densidades \\
& Transporte \\
\hline
\end{tabular}

DIMENSÃO
COGNITIVA

Representação mental Ordem simbólica

(Faria, 2010) a) Grafos multicamadas; hipergrafos

b) Ponderações nos vértices e arestas

\section{a) Grafos ponderados;}

b) Grafos direcionados entre pares de atividades complementares;

c) Nós ou arestas caracterizando conexões remotas

a) Agregação de elementos que compõem uma unidade informação em uma única entidade;

b) Conexões entre todos elementos que compõem uma unidade de informação;

c) Conexões remotas entre elementos com alguma afinidade cognitiva

a) Multigrafos

Curto prazo

Médio prazo

Longo prazo b) Camadas fixas e grafos direcionados

c) agregação de vértices e arestas iterativamente

d) 'thresholds', feedback positivo, pontos críticos e) polaridades com sentidos alternados
O Quadro 1 apresenta um framework multidimensional para se pensar a representação em múltiplas camadas e as estratégias de representação para cada tipo de 
elementos. Trata-se de uma ideia inicial, cujas possibilidades de representação não se restringem à essas sugeridas aqui. Elementos como os espaço públicos, vistos sob uma perspectiva puramente espacial podem ser representados por grafos simples, como os da Figura 1. Estes modelos podem ganhar novas nuances a partir de estratégias complementares, como a definição de raios para processamento das medidas, e a definição de valores de impedância para as arestas, que favoreçam ou desfavoreçam certos caminhos, como distância, tempo de deslocamento, hierarquia viária ou declividades.
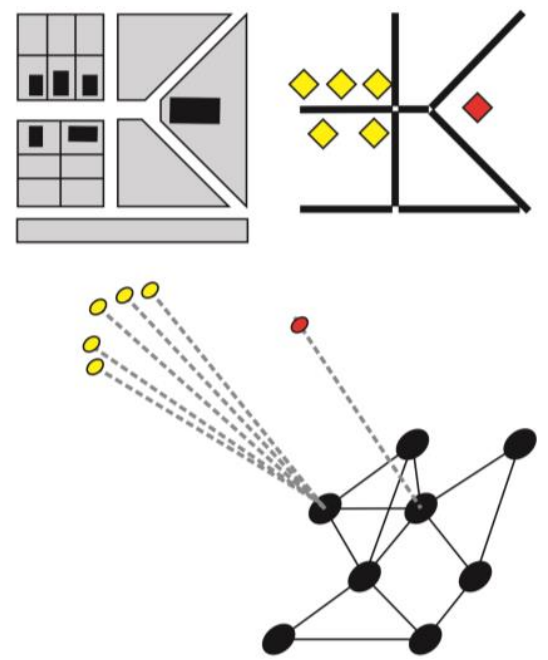

Figura 3. Grafo com duas camadas: espaços públicos e formas construídas (fonte: elaborada pelos autores).

O primeiro passo para estender esse tipo de representação estaria na inclusão das formas construídas, já eventualmente classificadas segundo tipos, ou segundo componentes de diferentes partes do tecido urbano, caractereizando uma dimensão objectual da forma urbana. Essa nova variável constitui uma segunda camada do grafo, conectado ao primeiro de forma particular. O mapograma da Figura 3 agora contém formas construídas, que, associadas ao mapa de segmentos anteriormente registrado no grafo inicial, constituem a segunda camada. A representação de elementos como formas construídas, parcelas, lugares, etc, pode ser feita pela inclusão de vértices que os representam, ou ainda por ponderação nos vértices do grafo dos espaços públicos, isto é, inserindo atributos que conferem um efeito de "carregamento" no grafo, que agora já não trata apenas da configuração espacial. Tal ideia já vem sendo explorada desde Krafta (1994).

Grafos ponderados são igualmente válidos para caracterizar a dimensão funcional. Esses atributos podem se referir a densidades urbanas, valor da terra, uso do solo, etc. É possível também trabalhar com a noção de grafos direcionados, isto é, especificar pares de atividades complementares, do tipo oferta e demanda ou origem e destino, de modo que se caracterizem relações de dependência funcional entre pares de nós específicos como em Krafta (1996) e Zechlinski (2013), e não do tipo "todos-com-todos", como normalmente se usa na análise configuracional. Grafos direcionados já foram explorados, por exemplo. Já para a representação do transporte, outro aspecto funcional das cidades, é preciso pensar em algum recurso que gere um efeito "atalho" no grafo, como, por exemplo, inserção de nós ou arestas que caracterizem conexões remotas entre pontos de parada do transporte público (Gil, 2014; Paroli, 2019).

Para a dimensão cognitiva, a representação deve buscar, de alguma forma, esquematizar sob a forma de grafos, as representações mentais do ambiente. Representações mentais dizem respeito ao conhecimento esquemático que cada indivíduo ou que uma comunidade tem do ambiente, ou seja, consistem nas informações ambientais cognitivamente estruturadas na mente humana. Não deixam de ser uma espécie de modelo espacial, portanto, também passível representação por grafos. Este tipo de descrição é praticamente inexplorado, com exceção do trabalho de Faria (2010). No processo de apreensão ambiental, elementos tendem a ser selecionados e agrupados, relações tendem a ser alteradas e distâncias distorcidas, de forma a facilitar o armazenamento na memória. Com base nesses processos é possível pensar em estratégias de representação das unidades de informação, que se referem ao conjunto de informação estruturada. Com base nos estudos de Faria (2010), para simular um efeito de agregação entre elementos que formam uma unidade informação, pode-se pensar em estratégias que envolvam: a) agregação dos vértices que a compõem em um único vértice; b) múltiplas conexões, do tipo todos-com-todos, entre os vértices que compõem uma mesma unidade de informação (exemplo: todos os vértices que 
representam uma rua ou um parque). Outro ponto que pode ser explorado é que a representação cognitiva, pode envolver conexões que não se dão por adjacência. Então, as arestas podem representar simplesmente algum tipo de afinidade cognitiva, criando uma ligação que aproxima ou que relaciona diretamente objetos que encontram-se fisicamente distantes.

Interessante destacar que não há apenas uma, mas sim várias abordagens possíveis dentro da ideia de redes com múltiplas camadas (Kivelä et al., 2014). Multigrafos, por exemplo, permitem representear conexões entre diferentes camadas, com o poder de associar atributos de diferentes tipos numa mesma operação algébrica, tomando por base a rede de espaços públicos. Assim, nós físicos remotos tornam-se adjacentes por força de conexões dadas pelo transporte público, ou polos de atividades são associados a hubs de transporte e, consequentemente, a espaços públicos (Figura 4a). De maneira semelhante, hipergrafos tratam de conectar vários nós por meio de uma mesma aresta; associados com outras camadas, transmitem propriedades agregadas (Figura 4b). Com base nessas definições, é possível pensar em maneiras/critérios para conectar diferentes camadas, pertencentes à distintas dimensões do framework delineado anteriomente.
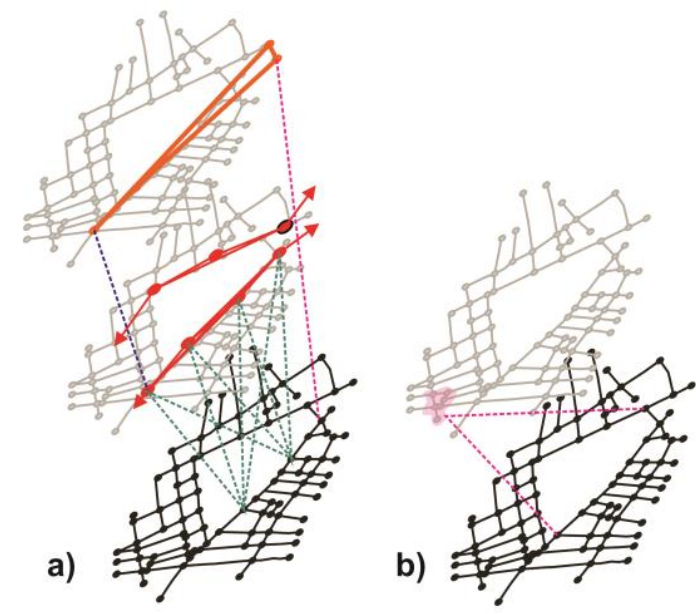

Figura 4. Exemplos de grafos multicamadas (fonte: elaborada pelos autores).

A noção de redes multicamadas também se presta a análises temporais (Kivelä et al., 2014), possibilitando a inclusão da dimensão temporal, isto é, a representação de processos de curto, médio e longo prazo, conforme exploramos a seguir.

\section{Morfologia urbana e análise do espaço- tempo}

Estudos da forma urbana baseados em grafos têm se focado principalmente na leitura de estado, isto é, na caracterização de algum particular aspecto do sistema urbano em dado momento. No entanto, é essencial avançar mais em pesquisas sobre como esses sistemas crescem, evoluem e se modificam ao longo do tempo e também sobre relações de interdependência entre variáveis e entre escalas (Batty, 2013). Nesta seção sugerimos uma maneira de passar da análise de estado à verificação de processo, mediante a inclusão da dimensão temporal na base descritiva por grafos multicamadas.

Análise clássica usando grafo envolve basicamente medidas de hierarquia, aglomeração e desempenho, entre outras possibilidades. Medidas de hierarquia envolvem classificar vértices ou arestas segundo suas posições comparadas segundo critérios de associação sistêmicas, tais como grau (conectividade), posição relativa (centralidade), ou distância relativa (acessibilidade). Estas medidas têm sido amplamente utilizadas em análise espacial urbana tanto aplicadas diretamente no grafo básico (espaço público) (Lima et al., 2017), quanto em grafos multicamadas, incluindo, então, forma construída, atividades ou valores cognitivos (Faria e Krafta, 2013). Medidas de aglomeração são, como o nome sugere, indicadores de distribuição espacial, tais como cluster, dispersão, densidade, diversidade, que podem incluir objetos, como formas construídas, equipamentos, etc., pessoas, atividades, usos do solo, renda e outras características socioeconômicas. Medidas de desempenho se aplicam aos casos em que privilégios ou dependências locacionais envolvendo normalmente usuários distribuídos e serviços concentrados, ou atividades cooperativas ou competitivas participantes de um mesmo sistema. Medidas características deste tipo são tensão, sinergia, estabilidade, desequilíbrio, oportunidade e convergência.

É visível aqui o potencial dos grafos multicamadas para incluir novas variáveis, constituindo aí um profícuo campo de pesquisa. Dados extraídos de diferentes plataformas digitais disponíveis tem sido testadas com sucesso em diferentes exercícios de análise utilizando grafos multicamadas ainda incipientes, mas 
sugerindo grandes possibilidades de desenvolvimento ulterior.

Os sistemas urbanos, e consequentemente os grafos representativos de suas redes sóciotécnicas mudam constantemente, propiciando seu exame segundo sua evolução. Mudanças ocorrem por modificação dos vértices localização, conteúdo, agentes, assim como das arestas - complementaridade funcional, estrutura cognitiva, base cultural, infraestrutura de comunicação, etc. ou por modificação de arestas, ao se introduzirem novas relações. Multigrafos podem ser utilizados não apenas para observação de estado, mas também para vereificação de processos. Vértices, atributos e relações que se modificam ao longo do tempo podem ser estudados por uma sequência de grafos, isto é, uma sequência de descrições de estado em um determinado momento. Para verificação de processos que se dão ao longo do tempo, é preciso adotar métodos de comparação de uma sequência de estados. Mudanças nos grafos podem ser verificadas, por exemplo, através de análises visuais, medidas-resumo ou índices baseados nas métricas de verificação de estado, que mostrem o impacto da adição de novos elementos (como em Strano et al., 2012) ou combinação de análises visuais com técnicas de estatística mais sofisticadas para verificação da evolução de padrões espaciais (com em Kirkley et al., 2018). Alometria é outro recurso que pode ser utilizado para verificação de processos evolutivos, ao permitir verificar relações de escala entre atributos - por exemplo, o quanto a centralidade aumenta à medida que um sistema aumenta de tamanho. A forma urbana é sujeita a relações alométricas à medida que a cidade cresce, uma vez que seus componentes crescem em diferentes ritmos e em diferentes proporções - como demonstram Krafta e Silva (2019), Shpuza (2014) e Strano et al. (2012).

O estudo da história das cidades tem mostrado que, não obstante terem origens e processos de desenvolvimento particulares, não parecem ser especiais - no sentido de se constituírem em singularidades - já que convergem para estruturas e organizações similares. Essa convergência tem sido atribuída a fatores geográficos - distância, fundamentalmente, e econômicos vantagens locacionais e de aglomeração. Dado que a distribuição dos componentes urbanos sobre o território envolve necessariamente separação e geração de afastamentos relativos, uma desigualdade fundamental, inerente ao fenômeno, se instala (distância) e é rapidamente socializada (vantagens comparativas), resultando em padrões de organização interna. Mesmo assumindo a robustez dessas teorias, apoiadas em evidências nas duas extremidades - a micro, onde cada agente supostamente buscaria minimizar distâncias e maximizar vantagens locacionais, e a macro, onde se verifica distribuições desparelhas de população e atividades econômicas, compondo uma paisagem hierarquizada, os caminhos trilhados desde as relações locais até cada macroestado são ainda nebulosos, para dizer o mínimo, e aí estaria possivelmente, em sua explicitação, a explanação definitiva do fenômeno urbano.

Aparentemente, cada cidade seguiria regras próprias para conectar agente social, com sua localização e elementos sociotécnicos com outros agentes, localizações e elementos sociotécnicos, envolvendo relações de produção e reprodução social particulares. Isso constituiria um vasto conjunto de regras locais diferenciadas umas das outras aplicadas a vastos conjuntos de agentes sociais ao longo de tempos extensos, cujo resultado esperado seria extrema diversificação. $O$ fato de haver convergências nos estados macro, isto é, homogeneização, mesmo que a um certo grau, sugere a existência de convertores de alguma espécie, sejam eles redutores de entropia (Haken e Portugali, 2015, Netto et al., 2017) ou invariáveis causais (Wolfran, 2020, Marchetti, 1990). Convertores agiriam no processo mediante regras que desfazem resultados de outras regras, evitando, dessa forma que a diversificação siga seu caminho ao infinito, e trazendo diferentes evoluções para macroestados convergentes. Regras, contrarregras de interação e invariantes causais sugerem caminhos inteiramente novos para a pesquisa urbana, para a qual os hipergrafos construídos a partir da morfologia urbana poderiam ser a base de representação.

É importante lembrar que interações entre agentes em escala espacial local ocorrem também em escala temporal imediata; a recorrência de práticas e a acumulação de seus efeitos no tempo e no espaço são os mecanismos básicos da mudança urbana. 
Paralelo e indissociável da prática recorrente ocorre outro fenômeno básico da mudança, poderia ser chamado de externalidade, isto é, a interferência, e consequente adaptação, de cada unidade de interação com as incontáveis demais interações ocorrendo nos mesmos tempos e lugares. Dessa maneira, não são apenas as práticas premeditadas e organizadas que contam na evolução dos sistemas urbanos, mas também as interferências não premeditadas e desorganizadas. A Figura 5 mostra uma sequência de situações, onde sobre uma base espacial, a princípio fixa, a quantidade de agentes, cada um desenvolvendo interações simples com outros dois, aumenta; as interações elementares passam a coexistir e criar interferências e adaptações umas com as outras, ampliando a articulação do sistema e promovendo conexões novas, entre células remotas. Esse processo tende a ser recursivo, isto é, formar um padrão, entretanto, a cada iteração podem ser esperadas mutações (novos agentes, novas interações) tornando o processo verdadeiramente complexo. De interações desse tipo - repetitivas, mas de curta duração, suscetíveis de causar e receber influências de outras ocorrendo simultaneamente pode-se esperar repercussões no espaço (propagação de efeitos locais, afetando partes maiores do sistema) e no tempo (adaptação dos diversos artefatos sociotécnicos que compõem a forma urbana e equipamentos complementares).

Tão importante quanto escalas temporais imediatas e recorrentes é considerar que interações, embora regulares e recorrentes, como ilustrado acima, não são contínuas. Com efeito, é fácil imaginar que, a um momento dado, alguns desses agentes, assim como suas interfaces com outros, estejam inativos, alterando a dinâmica espaçotemporal correspondente. Da mesma forma, a polaridade de determinados pontos da rede pode ser invertida, tal como ocorre com certos hubs urbanos, como escolas, que ora funcionam como atratores, ora como repulsores, concentradamente nos horários de entrada e saída, permanecendo inativos o resto do tempo (mesmo quando em funcionamento). Figura 6, extraída de Holme e Saramäky (2013), mostra um grafo temporal, a partir do qual um conjunto de medidas topológicas podem ser obtidas, à semelhança das medidas de centralidade dos grafos estáticos. Figura 7, também de Holme e Saramäky, é um grafo de intervalo, mostrando os intervalos de engajamento em interação de cada vértice.

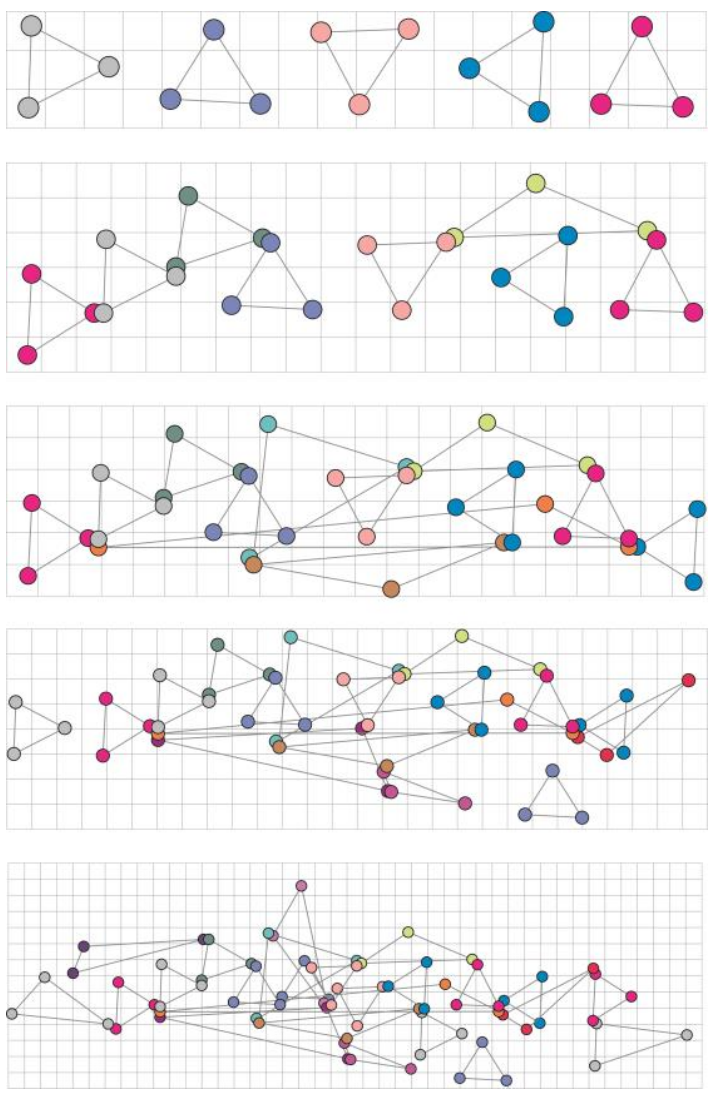

Figura 5. Sequência de interações entre agentes (fonte: elaborada pelos autores).

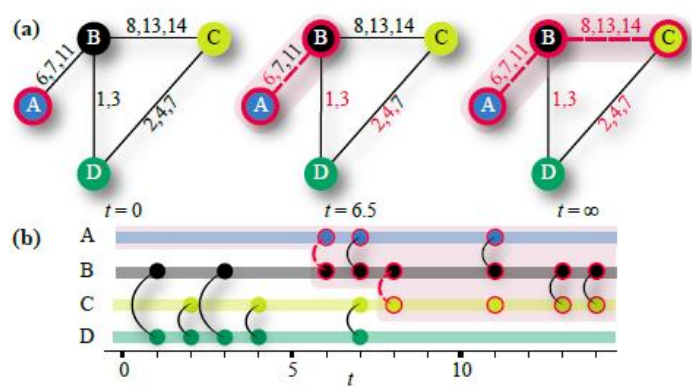

Figura 6. Grafo temporal (a) e painel de interação (b), sendo que a frequência de interação é registrada na forma de pesos atribuídos às arestas do grafo (fonte: Adaptado de Holme e Saramäky, 2013).

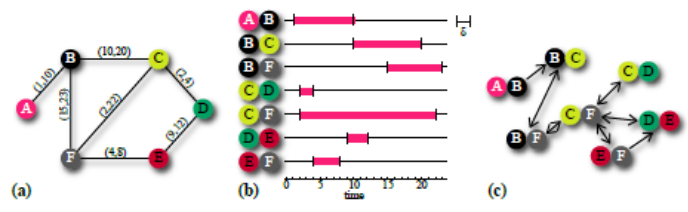

Figura 7. Grafo de transmissão, representando os intervalos de engajamento de uma rede temporal (fonte: Holme e Saramäky, 2013).

Inclusão da variável tempo e exploração de configurações mutáveis dos sistemas urbanos representa um campo de investigação capaz 
de, ao mesmo tempo, integrar a base de pesquisa em morfologia urbana com outras áreas de estudos urbanos e 'naturalizar' o acesso e uso de plataformas de dados existentes e sempre em evolução.

\section{Considerações finais}

A área de investigação conhecida como Morfologia Urbana tem desenvolvido uma base descritiva sólida e flexível para a cidade. Graças a isso tem ganho relevância e parece estar em posição de possibilitar a integração com outras áreas de estudo da cidade, mediante a ampliação de escopo, incorporação de variáveis e articulação de métodos. Como essa plataforma descritiva e analítica urbana tem sido constituída? No nosso entendimento, mediante os seguintes fatores:

a) Aproximação à ciência da complexidade se os fundamentos da ciência urbana, na sua origem, foram derivados de enunciados geográficos e econômicos, hoje os enunciados mais relevantes são os dos sistemas complexos. Não foi uma evolução original, já que a maioria das áreas do conhecimento realizaram a mesma trajetória, mas, dada a natureza conservadora da área, foi arrojada e surpreendente; representou uma mudança de perspectiva, até certo ponto radical, desde uma visão normativa e causal para outra evolucionária, instável e imprevisível do fenômeno urbano.

b) Apropriação de arcabouço teórico, modelos e métodos analíticos da física, principalmente da física estatística - a matematização da forma urbana parece ter sido um passo decisivo no caminho de quantificar, aferir, comparar, projetar, analisar e simular dinâmicas de mudança espacial. Esse poder de abstração torna tratáveis vários problemas inerentes à morfologia urbana, tais como quantidade e variedade de componentes e relações internas, explicitação de propriedades internas não visíveis, e o uso de modelos estatísticos mais sofisticados, entre outros.

c) 'Domesticação' de instrumentos computacionais - isto segue como complemento do anterior, visto que sistemas urbanos, ou mais simplesmente artefatos urbanos permaneceram intratáveis por tanto tempo justamente por possuírem enormes quantidades de componentes, derivando quantidades inimagináveis de possíveis configurações. Se a simples descrição de artefatos desse porte apresentam grandes problemas, parcialmente resolvidos pelos computadores, a sua análise permanece como um desafio, mesmo contando com o poder de processamento disponível hoje; grafos com algumas dezenas de milhares de vértices, contendo descrições de cidades normais, são comuns em análise de morfologia urbana hoje e demandam considerável esforço de processamento.

d) Formação de linguagem compatível com a das demais áreas da ciência - como síntese das apropriações acima referidas, a área tem desenvolvido uma linguagem - quer dizer, meios de representar o fenômeno urbano, semelhante às utilizadas em outras áreas de pesquisa científica. Isso tira a Morfologia Urbana da Sibéria, permite protocolos de transferência de procedimentos e falseamento de resultados interáreas e, como consequência, melhor aferição de qualidade e impacto.

Essas transformações pelas quais o campo da Morfologia Urbana vem passando, impulsionadas pelo desenvolvimento tecnológico e pela emergência do fenômeno big data, permitem vislumbrar possíveis expansões na investigaçao da forma urbana. $\mathrm{Na}$ dita fronteira do conhecimento já se verifa tendência a enfoques mais voltados à verificação de processos, isto é, que vão além da análise de estado. Os caminhos de pesquisa em Morfologia Urbana delineados neste artigo seguem nessa direção, a de cruzar fronteiras disciplinares, uma vez que buscam integrar variáveis que tradicionalmente eram tratadas de forma separada e, geralmente, sem uma perspectiva temporal. Espaço, equipamentos sociotécnicos, agentes e interação parecem constituir um conjunto indissociável de componentes e relações do cerne dos sistemas urbanos. Estender a pesquisa, incluindo esses processos, bem como entender suas interdependências é condição para o entendimento mesmo das cidades. 


\section{Notas}

${ }^{1}$ Artigo convidado para a seção temática "A nova ciência das cidades e a revolução dos dados".

2 www.urbanmorphologyinstitute.org , http://www.complexcity.info/2012/08/13/theurban-morphology-lab/, https://www.ucl.ac.uk/bartlett/casa/, https://cusp.nyu.edu/, https://miurban.uchicago.edu/ https://www.santafe.edu/, https://urbanstudies.uva.nl/

\section{Referências}

Alexander, C. (2015) A city is not a tree. Em: Mehaffy, M. (ed.). A City is not a Tree: 50th anniversary edition. Portland, Sustasis Press, edição do Kindle. [Original 1965]

Aleta, A., Moreno, Y. (2018). Multilayer Networks in a Nutshell. ArXiv. abs/1804.03488. Disponível em: https://doi.org/ 10.1146/annurev-conmatphys031218-013259.

Batty, M., Longley, P. (1988) The Morphology of Urban Land Use. Environment and Planning B, 15(4), 461488. Disponível em: https://doi.org/10.1068/b150461.

Batty, M. (2013) The new science of cities. Cambridge, MIT Press.

Boeing, G. (2017) OSMnx: New methods for acquiring, constructing, analyzing, and visualizing complex street networks. Computers, Environment and Urban Systems. 65, 126-139. Disponível em: https://doi.org/10.1016/j.compenvurbsys.2017.05. 004

Boeing, G. (2019) Street Network Models and Measures for Every U.S. City, County, Urbanized Area, Census Tract, and ZillowDefined Neighborhood. Urban Sci. 3(1), 28. Disponível em:

https://doi.org/10.3390/urbansci3010028

Caniggia, G. (1984) Lettura di una città: Como. Lomazzo, Italia, NewPress Edizioni.

Conzen, M. (ed.) (2004) Thinking about urban form: papers on urban morphology, 1932-1988 M.R.G. Conzen. Oxford, Peter Lang.

Faria, A. P. (2010) Análise configuracional da forma urbana e sua estrutura cognitiva. Tese de doutorado, Universidade Federal do Rio Grande do Sul, Programa de PósGraduação em Planejamento Urbano e Regional, Porto Alegre.
Faria, A.P., Krafta, R. (2013) Cognitive Structure: Urban Symbolic Order and Landmark Detection, Em: Palma, N. (org.) Sistemas Urbanos e Regionais. Santa Cruz do Sul, EDUNISC. Disponível em: http://hdl.handle.net/11624/1810 [Consultado em: 5 de junho de 2020].

Gil, J. (2014) Analyzing the configuration of multimodal urban networks. Geographical Analysis. 46(4), 368-391.

Greco, K. Seeing the city through data, seeing the data through the city. Em: Offenhuber, D., Ratti, C. (eds.) (2014). Decoding the city: Urbanism in the age of big data. Basel, Birkhauser, pp. 125-142.

Haken, H., Portugali, J. (2015) Information adaptation: The interplay between Shannon information and semantic information in cognition. Berlin, Springer.

Holme, P., Saramäky, J. (2013) Temporal Networks. Physics Reports. 519(3), 97-125. Disponível em: https://doi.org/10.1016/j.physrep.2012.03.001

Johnson, J. (2012) Cities: Systems of Systems of Systems. Em: Portugali, J., Meyer, H., Stolk, E., Tan, E. (eds.) Complexity theories of cities have come of age: An overview with implications to urban planning and design. Heidelberg, Springer, pp. 153-172.

Kirkley, A., Barbosa, H., Barthelemy, M., Ghoshal, G. (2018). From the betweenness centrality in street networks to structural invariants in random planar graphs. Nat Commun. 9, 2501. Disponível em: https://doi.org/10.1038/s41467-018-04978-Z

Kivelä, M., Arenas, al., Barthelemy, M., Gleeson, J., Moreno, Y., Porter, M. (2014) Multilayer networks. Journal of Complex Networks. 2(3), 203-271. Disponível em: https://doi.org/10.1093/comnet/cnu016.

Krafta, R. (1994) Modelling Intraurban Configurational Development, Environment and Planning B, 21(1), 67-82. Disponível em: https://doi.org/10.1068/b210067

Krafta, R. (1996) Modelling Intraurban Configurational Development, Environment and Planning B, 23(1), 37-48. Disponível em: https://doi.org/10.1068/b230037

Krafta, R., Silva, E. (2019) Self-Organized Criticality and urban form system dynamics with reference to a Brazilian city. Area Development and Policy. Disponível em: 
https://doi.org/10.1080/23792949.2019.1631 124

Lima, L, Krafta, R., Ribeiro, B. (2017) A distância como variável em modelos configuracionais no estudo da distribuição de atividades econômicas urbanas. URBE Rev.

Bras. Gest. Urbana, 9(2), 354-370.

Disponível em: https://doi.org/10.1590/21753369.009.002.ao14

Marshall, S., Gil, J., Kropf, K., Tomko, M., Figueiredo, L. (2018) Street network studies: from networks to models and their representations. Netw Spat Econ. 18(3), 735 749. Disponível em: https://doi.org/10.1007/s11067-018-9427-9.

Marchetti, C. (1990) Anthropological invariants in travel behaviour. Technological Forecasting and Social Change. 47, 75-88.

Netto, V.; Meirelles, J.V.; Pinheiro, M.; Lorea, H. (2017) Uma geografia temporal do encontro. Revista de Morfologia Urbana. 5(2), 85-101. Disponível em: http://revistademorfologiaurbana.org/index.p $\mathrm{hp} / \mathrm{rmu} /$ article/view/2. [Consultado em: $29 \mathrm{de}$ maio de 2020].

Netto, V., Meireles, J., Ribeiro, F. (2017). Social Interaction and the city: The effect of space in the reduction of entropy. Complexity. 2017, 6182503. Disponível em: https://doi.org/10.1155/2017/6182503.

Nicosia, V., Bianconi, G., Latora, V., Barthelemy, M. (2013) Growing multiplex networks. Phys. Rev. Lett. 111(5), 058701. Disponível em:

https://doi.org/10.1103/PhysRevLett.111.058 701.

Offenhuber, D., Ratti, C. (eds.) (2014). Decoding the city: Urbanism in the age of big data. Basel, Birkhauser.

Panerai, P., Castex, J., Depaule, J. (1997)

Formes Urbaines: de l'ilot a la barre. Marselha, France, Editions Parenthesis.

Paroli, E. (2019) A cidade como uma rede de interconexões sociais: uma abordagem configuracional. Dissertação de mestrado, Universidade Federal do Rio Grande do Sul, Programa de Pós-Graduação em Planejamento Urbano e Regional, Porto Alegre.

Porta. S., Rofè, Y., Vidoli, M. (2015) The city and the grid: building beauty at large scale. Em: Mehaffy, M. (ed.). A City is not a Tree: 50th anniversary edition. Portland, Sustasis Press, edição do Kindle.

Shpuza, E. (2014) Allometry in the syntax of street networks: Evolution of Adriatic and Ionian coastal cities 1800-2010. Environment and Planning B: Planning and Design. 41(3), p. 450-471. Disponível em: https://doi.org/10.1068/b39109.

Spinelli, J., Krafta, R. (1998) Configuração Espacial e Distribuição do Valor do Solo Urbano, Cadernos IPPUR, 12(2), 83-104. Disponível em: https://revistas.ufrj.br/index.php/ippur.

Strano, E., Nicosia, V., Latora, V., Porta, S., Barthelemy, M. (2012) Elementary processes governing the evolution of road networks. Sci Rep. 2(1), 296. Disponível em: https://doi.org/10.1038/srep00296.

Wasserman, S., Faust, K. (2009) Social network analysis: methods and applications. 18th ed. New York, Cambridge University Press.

Wolfran, S. (2020). Finally we may have a path to the fundamental theory of physics, and it's beautiful, Disponível em: https://writings.stephenwolfram.com [Consultado em: 29 de maio de 2020].

Zechlinski, A.P. (2013) Configuração e práticas no espaço urbano: uma análise da estrutura espacial urbana. Tese de doutorado. Universidade Federal do Rio Grande do Sul, Programa de Pós-Graduação em Planejamento Urbano e Regional, Porto Alegre. 


\section{Tradução do título, resumo e palavras-chave}

Urban Morphology and data revolution

Abstract. Urban Morphology is highly dependent on urban spatial data. For a long time, the difficulty in obtaining it constituted an obstacle to the development of researches in this area. However, more recently, the increase in the availability of data, the emergence of big data and the growing development of tools and information technologies, has been opening new possibilities to explore digitally the territory. It is not just the abundant availability of data and software to manipulate it, but new capabilities to integrate it, which encourages to cross-disciplinary boundaries. This article focuses on the relationship between the field of urban morphology, characterized by well-defined outlines in terms of what kind of data is required, and what has been done at the frontier of knowledge, where data of different types are combined. As a contribution, this work points out new insights for future research, showing how urban morphology can benefit from this data revolution, especially by using new descriptive and analytical mechanisms. In this sense, we outline a multilayer graph approach, which is still little explored in urban studies, despite showing potential for a) multidimensional descriptions of urban form; b) explorations of space-time assessments.

Keywords: urban morphology, big data, multilayer networks, temporal networks

Editor responsável pela submissão: Vinicius de Moraes Netto.

Licenciado sob uma licença Creative Commons. 